\title{
Binding energies of quantum dipole in plane
}

\author{
Eugene A. Koval ${ }^{1, \star}$ and Oksana A. Koval ${ }^{2,3, \star \star}$ \\ ${ }^{1}$ Bogoliubov Laboratory of Theoretical Physics, Joint Institute for Nuclear Research, Dubna, Moscow Re- \\ gion, Russia \\ ${ }^{2}$ Dzhelepov Laboratory of Nuclear Problems, Joint Institute for Nuclear Research, Dubna, Moscow Region, \\ Russia \\ ${ }^{3}$ Obukhov Institute for Atmospheric Physics, Moscow, Russia
}

\begin{abstract}
We propose a numerical algorithm based on a discrete variable representation and shifted inverse iterations and apply it to for the analysis of the bound states of edge dislocation modelled by a quantum dipole in a plane. The good agreement with results of recent papers of Amore et al [J. Phys. B 45, 235004 (2012)] was obtained. The error estimates of the previous results of low-lying states energies of other authors were not known due to limitations of the variational approaches and this paper fills this gap presenting calculated low-lying bound states energies by non-variational technique. The probability densities of low-lying states were calculated.
\end{abstract}

\section{Introduction}

The aim of this paper is study the low-lying bound states of the straight edge dislocation in solids. The interaction of electron with such dislocation deformation, oriented along the $Z$ axis, is modelled by anisotropic potential of the form [1]:

$$
V(\rho, \phi)=p \frac{\cos (\phi)}{\rho}
$$

where $\rho$ and $\phi$ are the polar coordinates, defined in the $X Y$ plane, $p$ is the strength of the dipole potential. It can be realized as a dipole built by bringing two infinite line charges of opposite sign close together [1].

An anisotropy of an interaction strongly affects the system properties, as it was revealed in physics of ultracold atoms and polar molecules [2], Rydberg atoms in external fields [3], excitons in semiconductor heterostructures [4], producing exotic stable configurations in ultracold gases [5].

Due to the nonseparability of the potential (1) the quantitative analysis is difficult because traditional analytical techniques are no longer applicable and effective numerical methods are required for solving of the full Shrödinger equation. We propose such a numerical algorithm and numerically solve the corresponding two-dimensional (2D) Schrödinger equation [1]:

$$
\left[-\frac{\hbar^{2}}{2 \mu}\left(\frac{1}{\rho} \frac{\partial}{\partial \rho}\left(\rho \frac{\partial}{\partial \rho}\right)+\frac{1}{\rho^{2}} \frac{\partial^{2}}{\partial \phi^{2}}\right)+p \frac{\cos (\phi)}{\rho}\right] \Psi(\rho, \phi)=E \Psi(\rho, \phi) .
$$

\footnotetext{
$\star$ e-mail: e-cov@yandex.ru

${ }^{\star}$ e-mail: kov.oksana20@gmail.com
} 
In the units of length $\frac{\hbar^{2}}{2 m p}$ and energy $\frac{2 m p^{2}}{\hbar^{2}} 2 \mathrm{D}$ SE reads:

$$
\left[-\left(\frac{1}{\rho} \frac{\partial}{\partial \rho}\left(\rho \frac{\partial}{\partial \rho}\right)+\frac{1}{\rho^{2}} \frac{\partial^{2}}{\partial \phi^{2}}\right)+\frac{\cos (\phi)}{\rho}\right] \Psi(\rho, \phi)=\epsilon \Psi(\rho, \phi) .
$$

The potential is invariant under reflection about the $X$ axis $V(\rho, \phi)=V(\rho,-\phi)$, and due to this symmetry of the potential the bound states are either even $\Psi(\rho, \phi)=\Psi(\rho,-\phi)$ or odd $(\Psi(\rho, \phi)=$ $-\Psi(\rho,-\phi))$. The potential is illustrated on Fig. 1, demonstrating that for $p>0$ the bound states are allowed in $x<0$ region.

As it was noted by Dasbiswas et al [1], from the initial Landauer estimate (-0.102 arb.u.) [6] of ground state energy (GSE) there were several attempts of other authors [6-11] with different basis functions to calculate it more precisely within variational approach. The comparison of results of several real-space discretization methods (RSDM), such as the biconjugate gradient method, the Jacobi-Davidson algorithm and Arnoldi-Lanczos algorithm, was shown in Ref. [1]. In the paper [1] it was noted, that the RDSM methods are preferable for low-lying states than variational studies, but the best GSE value (-0.139 arb.u.) was calculated by RDSM only with 2\% accuracy. Amore et al [12] showed, that variational technique for Slater-type orbitals are converged faster and seems to be more accurate for the GSE value, than the Coulomb basis set. It was also noted in Ref. [12], that, possibly, bad convergence of variational studies for low-lying states comes from limited accuracy of the method due to not complete basis of the basis function set. Handy et al [13] proved this statement, expanding the wave function over a complete basis with the help of an orthogonal polynomial projection quantization analysis, which substantially decreased the needed for convergence variational parameters number.

\section{Edge dislocation bound states}

One of the remained problem is that within variational approach there is no technique for estimating the accuracy of the results, that depend on the appropriate choice of the form of the trial function. We fill this gap employing the proposed numerical algorithm for solving the $2 \mathrm{D}$ Schrödinger equation. It is based on the method of shifted inverse iterations and the variation of the discrete-variable method, proposed in the paper of Melezhik [14] for a solution of the multichannel scattering problem and

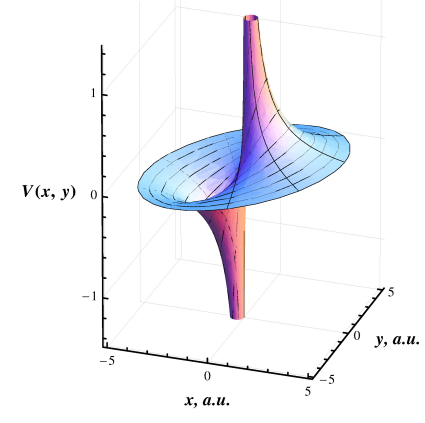

Figure 1. The potential surface of the deformation potential (1). 
applied for study of the 2D scattering of two unpolarized dipoles $[15,16]$ and the 2D Hydrogen atom in a tilted magnetic field $[17,18]$.

The eigenfunctions $\xi_{m}(\phi)=\frac{(-1)^{m}}{\sqrt{2 \pi}} e^{i m \phi}$ of the operator $h^{(0)}(\phi)=\frac{\partial^{2}}{\partial \phi^{2}}$ are used as a basis of functions for wave function expansion over angular variable.

Wave function is expanded as follows:

$$
\Psi(\rho, \phi) \approx \frac{1}{\sqrt{\rho}} \sum_{m=-M}^{M} \sum_{j=0}^{2 M} \xi_{m}(\phi) \xi_{m j}^{-1} \psi_{j}(\rho)=\frac{1}{(2 M+1) \sqrt{\rho}} \sum_{j=0}^{2 M} \sum_{m=-M}^{M} e^{i m\left(\phi-\phi_{j}\right)} \psi_{j}(\rho),
$$

where $\xi_{m j}^{-1}=\frac{2 \pi}{2 M+1} \xi_{j m}^{*}=\frac{\sqrt{2 \pi}}{2 M+1} e^{-i m\left(\phi_{j}-\pi\right)}-$ is the inverse mathrix to the square matrix $(2 M+1) \times$ $(2 M+1) \xi_{j m}=\xi_{m}\left(\phi_{j}\right)$, that is defined on the uniform angular grid $\phi_{j}=\frac{2 \pi j}{2 M+1}$ (where $\left.j=0,1, \ldots, 2 M\right)$. In the angular grid's nodes $\phi_{j}: \Psi\left(\rho, \phi_{j}\right) \approx \psi_{j}(\rho) / \sqrt{\rho}$.

In representation (4) 2D Schrödinger equation transforms in the system of $(2 M+1)$ coupled second-order differential equations:

$$
-\frac{\partial^{2}}{\partial \rho^{2}} \psi_{j}(\rho)-\frac{1}{4 \rho^{2}} \psi_{j}(\rho)+\sum_{j^{\prime}=0}^{2 M} V_{j j^{\prime}} \psi_{j^{\prime}}(\rho)-\frac{1}{\rho^{2}} \sum_{j^{\prime}=0}^{2 M} h_{j j^{\prime}}^{(0)} \psi_{j^{\prime}}(\rho)=\epsilon \psi_{j}(\rho) .
$$

The nondiagonal matrix of $h^{(0)} \equiv \frac{\partial^{2}}{\partial \phi^{2}}$ operator is defined by the expression:

$$
h_{j j^{\prime}}^{(0)}=-\sum_{j^{\prime \prime}=-M}^{M} j^{\prime \prime 2} \xi_{j j^{\prime \prime}} \xi_{j^{\prime \prime} j^{\prime}}^{-1}
$$

Since, expression $\psi_{j} / \sqrt{\rho}$ must be finite at $\rho=0$, radial components $\psi_{j}$ vanish at $\rho \rightarrow 0$ and left boundary condition reads:

$$
\psi_{j}(0)=0 .
$$

The bound state wave function natural decay in the infinity impose the right boundary condition:

$$
\psi_{j}(\rho \rightarrow \infty) \rightarrow 0 \quad(j=0,1, \ldots, 2 M) .
$$

To discretize the boundary problem $(5,7,8)$ we employ nonuniform grid over radial variable: $\rho_{n}=$ $\rho_{N} t_{n}^{2}, \quad(n=1,2, \ldots, N)$, which nodes are mapped $\rho_{n} \in\left[0, \rho_{N} \rightarrow \infty\right]$ on a uniform grid $t_{n} \in[0,1]$. The seven-point finite difference approximation of six-order accuracy is used for the derivative. The matrix eigenvalue problem is solved by the method of inverse iterations with shift. An obtained on each iteration matrix problem is tackled with the matrix modification of the sweep algorithm for band matrix.

The algorithm advantages are recapped below. Comparing with variational studies there is the approximation error estimate of the used wave function expansion (4), that indicates a fast convergence over the number of angular grid nodes. Obtained matrices has band structure, which allows optimal resource using. The fast convergence over inverse iterations is confirmed by the small average inverse iterations number $\approx 10$, needed for the first six digits remain stable.

With the help of the proposed numerical scheme we calculated up to 6 significant digits the binding energies of a quantum dipole model in 2D and eigenfunctions of the five low-lying even bound states and improved the results accuracy of the previous studies [1], proving recent results [12, 13]. In Table 1 the comparison of the calculated by us with 6-digit accuracy binding energies $\epsilon_{b}$ of five $(n=1-5)$ low-lying even bound states (3rd column) with the results of other authors, obtained with 
Table 1. The comparison of the calculated by us with 6-digit accuracy binding energies $\epsilon_{b}$ of five $(n=1-5)$ low-lying even bound states (3rd column) with the results of other authors, obtained with variational techniques over 2D Coulomb eigenfunctions [1] and over Slater function [12] (the unit of energy is $\frac{2 m p^{2}}{\hbar^{2}}$ ).

\begin{tabular}{|cccc|}
\hline$n$ & $\epsilon_{b}[1]$ & $\epsilon_{b}[12]$ & $\epsilon_{b}$ \\
\hline 1 & 0.0970 & 0.137741 & 0.137748 \\
2 & 0.0328 & 0.041152 & 0.041158 \\
3 & 0.0221 & 0.019967 & 0.019973 \\
4 & 0.0167 & 0.011852 & 0.011858 \\
5 & 0.0119 & 0.009747 & 0.009747 \\
\hline
\end{tabular}

Table 2. The dependence of the binding energies $\epsilon_{b}$ (in the units $\frac{2 m p^{2}}{\hbar^{2}}$ ) of five $(n=1-5)$ low-lying even bound states on the number of angular-grid points $M$.

\begin{tabular}{|c|lllll|}
\hline & \multicolumn{5}{|c|}{$\epsilon_{b}$} \\
$M$ & $n=1$ & $n=2$ & $n=3$ & $n=4$ & $n=5$ \\
\hline 6 & 0.137671 & 0.040562 & 0.019291 & 0.011255 & 0.008853 \\
8 & 0.137747 & 0.041110 & 0.019845 & 0.011702 & 0.008571 \\
10 & 0.137748 & 0.041156 & 0.019957 & 0.011825 & 0.009115 \\
20 & 0.137748 & 0.041159 & 0.019974 & 0.011859 & 0.009747 \\
40 & 0.137748 & 0.041159 & 0.019974 & 0.011859 & 0.009747 \\
\hline
\end{tabular}

variational techniques over 2D Coulomb eigenfunctions [1] and over Slater function [12] is presented. The analysis of Table 1 shows the obtained good agreement with papers [1, 12].

In Table 2 the dependence of the binding energies $\epsilon_{b}$ (in the units $\frac{2 m p^{2}}{\hbar^{2}}$ ) of five $(n=1-5)$ low-lying even bound states on the number of angular-grid points $M$ confirms the fast convergence over angular grid $(M \approx 20$ is enough for 6-digit accuracy).

The corresponding calculated probability densities are presented on Fig. 2. The analysis of Fig. 2 demonstrates, that their shapes correspond to the anisotropic potential form.

\section{Conclusion}

The bound states of edge dislocation modelled by a quantum dipole in a plane are numerically investigated with the help of the proposed numerical algorithm based on a discrete variable representation and shifted inverse iterations. We reproduced the results of other authors [12, 13], obtained by variational studies, and calculated low-lying states energies with 6-digit accuracy. The obtained probability densities of low-lying states correspond to the anisotropic potential form.

The authors acknowledge the support by the Russian Foundation for Basic Research, Grant No. 19-32-80003.

\section{References}

[1] K. Dasbiswas, D. Goswami, C.D. Yoo, A.T. Dorsey, Physical Review B 81, 064516 (2010) 

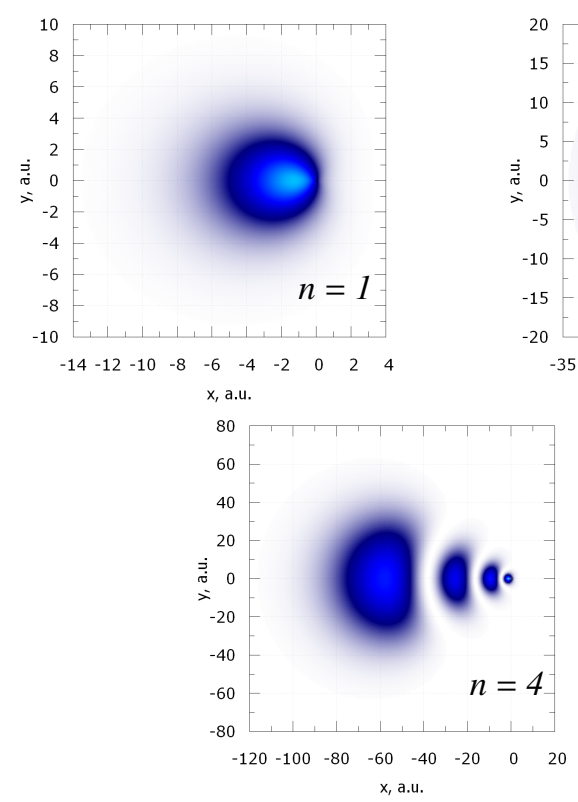
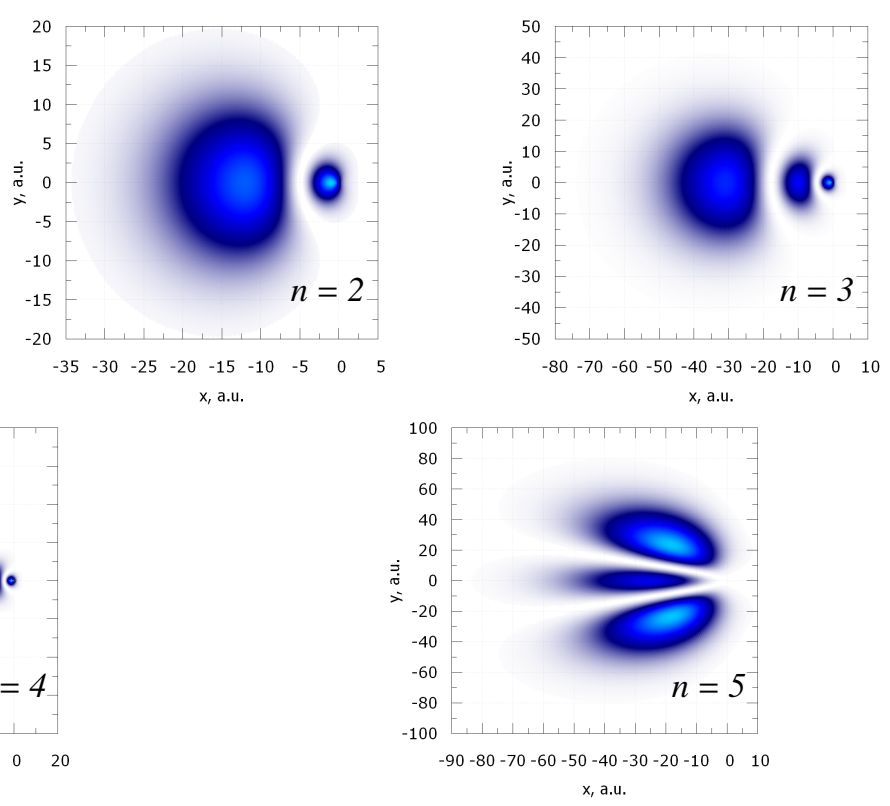

Figure 2. Probability density plots of five $(n=1,2, \ldots, 5)$ low-lying even bound states. Dark blue regions correspond to low and bright blue ones to high densities. The quantities are given in atomic units.

[2] T. Lahaye, C. Menotti, L. Santos, M. Lewenstein, T. Pfau, Rep. Prog. Phys. 72, 126401 (2009)

[3] A. Browaeys, D. Barredo, T. Lahaye, Journal of Physics B: Atomic, Molecular and Optical Physics 49, 152001 (2016)

[4] V.B. Timofeev, A.V. Gorbunov, Physica Status Solidi (C) 5, 2379 (2008)

[5] I. Ferrier-Barbut, H. Kadau, M. Schmitt, M. Wenzel, T. Pfau, Phys. Rev. Lett. 116, 215301 (2016)

[6] R. Landauer, Physical Review 94, 1386 (1954)

[7] P. Emtage, Physical Review 163, 865 (1967)

[8] V. Nabutovskii, B. Shapiro, JETP Lett., 26, 473 (1977)

[9] V. Slyusarev, Fiz. Met. Metalloved. 58, 877 (1984)

[10] I. Dubrovskii, Low Temperature Physics 23, 976 (1997)

[11] J.L. Farvacque, P. Francois, Physica Status Solidi (b) 223, 635 (2001)

[12] P. Amore, F.M. Fernández, Journal of Physics B: Atomic, Molecular and Optical Physics 45, 235004 (2012)

[13] C. Handy, D. Vrinceanu, Journal of Physics B: Atomic, Molecular and Optical Physics 46, $115002(2013)$

[14] V.S. Melezhik, Journal of Computational Physics 92, 67 (1991)

[15] E.A. Koval, O.A. Koval, European Physical Journal Web of Conferences 173, 06008 (2018)

[16] E.A. Koval, O.A. Koval, V.S. Melezhik, Physical Review A 89, 052710 (2014)

[17] E.A. Koval, O.A. Koval, Journal of Experimental and Theoretical Physics 125, 35 (2017), [Pis'ma Zh. Eksp. Teor. Fiz. 152, 45 (2017)]

[18] E.A. Koval, O.A. Koval, Physica E 93, 160 (2017) 